Methods Participants were recruited from a GBM sexual health clinic and completed a one-time 60-minute education session with a peer health educator. This included condom and lubricant information and condom application skills practice. Between 09/2018-02/2019, each participant completed a baseline survey prior to intervention and again three months later, which were compared using paired t-tests.

Results A total of 24 HIV-negative participants received the intervention: average age was 27.8 years $(\mathrm{SD}=3.53)$ and $52 \%$ identified as non-white. The intervention was highly acceptable: $87 \%$ liked it and 91\% would recommend it to others. At 3-month follow-up, participants agreed the intervention increased: knowledge about using lubricants with condoms (83\%), condom use skills (78\%), and condom use confidence (70\%). At baseline, few participants used daily pre-exposure prophylaxis (PrEP, 17\%); post-intervention, 6 PrEP-naïve participants reported initiating PrEP (32\%). Overall, condom use frequency during anal sex with male partners did not change (51\% baseline versus $58 \%$ post-intervention, $\mathrm{p}=0.41)$. However, among non-PrEP users, condom use frequency significantly increased during receptive anal sex $(61 \%$ baseline versus $78 \%$ post-intervention, $\mathrm{p}=0.04)$ and marginally increased during insertive anal sex (24\% baseline versus $48 \%$ post-intervention, $\mathrm{p}=0.11$ ).

Conclusion The adapted FOF intervention was highly acceptable to ethnoracially diverse GBM in Vancouver. A third of participants initiated PrEP within 90 days. Among participants not using PrEP, the intervention effectively increased condom use during receptive anal sex, when HIV acquisition is most likely. This low-cost intervention demonstrates promise for increasing prevention practices among GBM attending STI clinics in Vancouver.

Disclosure No significant relationships.

\section{P323 UNEXPECTED RISING TREND OF SEXUALLY TRANSMITTED INFECTION IN BANGKOK, THAILAND}

Pannee Chaiphosri, Alisra Tattakorn*, Kanokrat Lerdtriphop. BMA, Health Department, Bangkok, Thailand

10.1136/sextrans-2019-sti.434

Background Sexually transmitted infection (STI) remain a major public health challenge in Thailand, particularly Bangkok. STI control is important for HIV prevention. With a commitment to United Nation's Fast-Track cities to end AIDS in Bangkok by 2030, situation of STI should be monitored to assess effectiveness of HIV prevention efforts. This study aimed to examine trends of STI in Bangkok from 2013 to 2017 using case reporting data and periodic surveys.

Methods Data were obtained from the National Notifiable Disease Surveillance Report (506) and Routine Integrated HIV Information System (RIHIS) from nine STI clinics in Bangkok between 2013 and 2017.

Results Bangkok is facing a rise in STI cases, especially among young people, with $40 \%$ of all STI cases occurring in 15-24 years. From 2013 to 2017, the rates of reported STI in Bangkok increased eight folds from 11.8 cases per 100,000 population to 90 cases per 100,000 , while the national rate rose four folds (18.5 in 2013 to 67.2 in 2017). In 2017, syphilis was the most commonly reported disease in Bangkok with two times the national rate and particular common among men who have sex with men (MSM).

Conclusion Prevalence of STI in Bangkok was rising and much higher than the national scale. MSM account for a disproportionately large burden of STIs particularly syphilis, which are consistent with high prevalence of HIV infection among MSM. Weakening of STI control may undermine HIV prevention efforts. Strengthening STI control in Bangkok are urgently needed.

Disclosure No significant relationships.

\section{P324 HOW DO THE PSYCHOSOCIAL CHARACTERISTICS OF WOMEN ATTENDING SEXUAL HEALTH SERVICES DIFFER FROM THOSE ATTENDING PRIMARY CARE?}

${ }^{1}$ Natalie Edelman*, ${ }^{2}$ Jennifer Whetham, ${ }^{3}$ Abi Gersten, ${ }^{4}$ Catherine Mercer, ${ }^{5}$ Richard De Visser, ${ }^{6}$ Chris Jones, ${ }^{6}$ Stephen Bremner, ${ }^{6}$ Jackie Cassell. ${ }^{1}$ University of Brighton, School of Health Sciences, Westlain House, Brighton and Hove, UK; ${ }^{2}$ Brighton and Sussex University Hospitals Trust, Brighton and Hove, UK; ${ }^{3}$ Pavilion Surgery, Brighton and Hove, UKi ${ }^{4}$ University College London, Institute for Global Health, London, UK; ${ }^{5}$ University of Sussex, School of Psychology, Brighton and Hove, UK; ${ }^{6}$ Brighton and Sussex Medical School, Primary Care and Public Health, Brighton and Hove, UK

\subsection{6/sextrans-2019-sti.435}

Background Women attending specialist sexual health and contraception clinics (SHAC) are younger and more likely to report substance use and sexual risk behaviours than those attending Primary Care (PC). A broader analysis of psychosocial differences between these populations may improve our understanding of the wider determinants of sexual risk and morbidity and support the development of psychosocial interventions for use in specialist settings. We therefore explored which psychosocial factors were associated with recruitment site.

Methods Psychosocial question responses were compared from a cross-sectional survey of convenience-sampled women aged 16-44 years attending PC (Primary Care) vs SHAC services in the city of Brighton and Hove, UK. Multivariable logistic regression was used to identify which psychosocial factors predicted attendance in SHAC versus PC.

Results 1238 (70\%) eligible women completed a questionnaire in a PC setting and 532 (30\%) women in a SHAC service. After controlling for age, several psychosocial factors predicted SHAC compared to PC attendance. These included: living in rented accommodation (adjusted odds ratio $(\mathrm{aOR})=1.70,95 \%$ confidence interval (CI):1.20-2.40), being a cigarette smoker $(\mathrm{aOR}=1.32,95 \% \mathrm{CI}: 1.00-1.75)$, disagreement that 'having a partner at all times is important to me' $(\mathrm{aOR}=2.24,95 \%$ CI:1.69-2.97) emotional dissatisfaction with most recent relationship $(\mathrm{aOR}=1.51,95 \% \mathrm{CI}: 1.15-1.99)$ and little or no functional social support (e.g. help with chores and meals) (aOR=1.83,95\%CI:1.21-2.78).

Conclusion Findings suggest that women attending SHAC may be more likely to experience lack of support and dissatisfaction with sexual and other relationships, and may be more likely to be in rented or other insecure housing compared with those attending primary care settings. Thus, the potential impact of broader life circumstances on sexual risk may be 
worthy of discussion during clinical and health advisor consultations.

Disclosure No significant relationships.

\section{P326 SYNDEMIC PATTERNS OF RISK FOR SEXUALLY TRANSMITTED INFECTIONS}

${ }^{1}$ Ashley Hill*, ${ }^{2}$ Tamika Gilreath, ${ }^{3}$ Maria Perez-Patron, ${ }^{4}$ Brandie Taylor. ${ }^{1}$ Texas AandM University, College Station, USA; ${ }^{2}$ Texas AandM University, Transdisciplinary Center For Health Equity Research, College Station, USA; ${ }^{3}$ Texas AandM University, Epidemiology and Biostatistics, College Station, USA; ${ }^{4}$ Temple University, Epidemiology and Biostatistics, Philadelphia, USA

10.1136/sextrans-2019-sti.436

Background Syndemics theory proposes that diseases may cluster and intensify based on multiple interacting factors. Few studies have compared methods to identify syndemics related to sexually transmitted infection (STI) risk. The purpose of this study is to compare the use of a composite scoring method and latent class analysis to identify syndemics of STI risk.

Methods Youth were aged 18-25 who participated in the National Health and Nutrition Examination Survey, 2011$2014(\mathrm{~N}=1,803)$. Syndemic composite scoring was tabulated by an accumulation of indicators by gender, and latent class analysis (LCA) was conducted to extract classes of risk based on indicators reported by gender. The outcome, STIs, was defined as a determinant result for Chlamydia trachomatis, Herpes Simplex Virus type-2, or HIV. The odds of STI were calculated using the patterns of risk by sex adjusted for demographic and poverty indicators.

Results Composite score results suggest that males with an accumulation of 3 to 5 syndemic indicators (AOR: $2.10 \mathrm{CI}$ 95\% 1.0-4.2) and 6 or more indicators (AOR: 2.84 CI 95\% 1.2-6.7) had an increased odds of STI. Similarly, females with 6 or more indicators (AOR: 3.20 CI 95\% 1.7-6.0) had increased odds of STI. The LCA suggested that men with the highest probability of smoking and sexual risk behaviors were at increased odds of STI (AOR: 2.42 CI 95\% 1.1-5.4), while women exhibit a syndemic of depression, smoking, drug use and sexual risk behaviors (AOR: 2.19 CI 95\% 1.2-3.8).

Conclusion The co-occurrence of mental health,substance use and smoking were important indicators of STI risk in women. LCA was able to determine indicators that co-occurred in men and women and sexual risk behaviors that differed by gender, while the syndemic scoring show an accumulation of indicators increased STI risk.

Disclosure No significant relationships.

\section{P327 PATTERN OF SEXUALLY TRANSMITTED INFECTIONS IN THE INTERIOR PART OF SINDH PROVINCE OF PAKISTAN}

${ }^{1}$ Mour Khan*, ${ }^{2}$ Shahnawaz Bhutto, ${ }^{3}$ Nadia Agha, ${ }^{4}$ Erum Junejo. ${ }^{1}$ Shah Abdul Latif University and CMC Larkana, Sociology, Khairpur, Pakistan; ${ }^{2}$ Shah Abdul Latif University and CMC Larkana, Economics, Khairpur, Pakistan; ${ }^{3}$ Shah Abdul Latif University, Sociology, Khairpur, Pakistan; ${ }^{4}$ Chandka Medical College and SMBB Medical University, Dermatology, Larkana, Pakistan

\subsection{6/sextrans-2019-sti.437}

Background Sexual Transmitted infections (STls) are widespread in Pakistan and have not been fully documented. A hospital based prospective study was carried out to determine the incidence and clinical pattern of various types of STls in general population of Larkana division and its surrounding cities.

\section{Methods}

Patients and methods This study was conducted at male STD Clinic in the Department of Dermatology Shaheed Mohtarma Benazir Bhutto Medical University (SMBBMU) hospital Larkana. A total of 4288 cases were seen in this semi urban area. A structured questionnaire presenting the details of syndromic diagnosis was filled during the examination of each patient. For our ease and understanding, these patients were also separated on the basis of clinical and etiological grounds. Results Among 4288 patients, 3947 had the history of extra marital sexual contact and simultaneously had developed clinical signs of STls. Majority of patients had the history of heterosexual contact with different partners. According to syndromic diagnosis: 1930 patients had genital ulcer (including herpes genitals) with or without skin manifestations, 690 had urethral discharge, 431 had genital warts, 349 had lesions other than STls related, 304 had more than one syndrome, 193 had scrotal swelling, 46 had inguinal baboo, 3 were human immunodeficiency virus (HIV) positive and 1 had ophthalmia neonatorium.

Conclusion It is concluded that 5Tls are the growing concern and a public health problem in the central part of 5 indh province of this muslim state and syphilis exhibited the highest level of concentration followed by gonorrhea and so on. Community needs to be educated for adopting the safe sex measures to prevent the STls.

Disclosure No significant relationships.

\section{P328 SEXUAL HEALTH CARE: PROFESSIONAL DEVELOPMENT FOR RURAL PRACTITIONERS}

Siobhan Bourke*, Jane Tomany. University of Melbourne, Department of Rural Health Centre for Excellence in Rural Sexual Health, Wangaratta, Australia

\subsection{6/sextrans-2019-sti.438}

Background Rural doctors have limited accessible professional development, barriers include time, travel, expense and relief staffing issues. Sexual Health Care education opportunities were lacking, so we have provided online, free, accredited education for rural practitioners that was relevant to their practice. The online education modules, which are unique, use a rural lens when addressing sexual health care needs in practice in rural communities.

Methods The Centre for Excellence in Rural Sexual Health instigated the production of online learning modules. The first 8 were launched in 2016 and a further two have been launched since. Each module is evaluated by the participant upon completion. The modules cover practical topics in sexual health care ranging from sexual history taking, partner notification in a small community to avoiding assumptions in sexual health care.

Results 326 modules have been completed. The most popular modules were 'Introduction to rural sexual health care', 'Cornerstones of sexual health care' and 'STI treatment and management modules'. These modules are compulsory for a General Practitioner to complete if he or she is completing them as part of a large professional development activity (known as an Active Learning Module). The rest of the modules are free choice; with the most popular module being 